\title{
A NON-COMMUTATIVE SOBOLEV ESTIMATE AND ITS APPLICATION TO SPECTRAL SYNTHESIS
}

\author{
M. K. VEMURI
}

\begin{abstract}
In [M. K. Vemuri, Realizations of the canonical representation, Proc. Indian Acad. Sci. Math. Sci., 118 (2008), 115-131], it was shown that the spectral synthesis problem for the Alpha transform is closely related to the problem of classifying realizations of the canonical representation (of the Heisenberg group). In this paper, it is shown that discrete sets are sets of spectral synthesis for the Alpha transform.
\end{abstract}

\section{Introduction}

For $p \in[1, \infty]$, let $S^{p}$ denote the Schatten class of $p^{\text {th }}$ power traceable operators on $L^{2}(\mathbb{R})$. For $x, y \in \mathbb{R}$, let $T_{x}$ and $M_{y}$ denote the translation and modulation operators on $L^{2}(\mathbb{R})$, i.e. for $s \in L^{2}(\mathbb{R})$

$$
\begin{aligned}
\left(T_{x} s\right)(t) & =s(t+x) \quad \text { and } \\
\left(M_{y} s\right)(t) & =e^{2 \pi i y t} s(t) .
\end{aligned}
$$

If $\left(x_{1}, y_{1}\right) \in \mathbb{R}^{2}$ and $X \in S^{p}$, set

$$
\left(x_{1}, y_{1}\right) \cdot X=T_{y_{1}} M_{-x_{1}} X M_{x_{1}} T_{-y_{1}} .
$$

If $q \in L^{1}\left(\mathbb{R}^{2}\right)$ and $X \in S^{p}$, set

$$
q \cdot X=\iint q\left(x_{1}, y_{1}\right)\left(x_{1}, y_{1}\right) \cdot X d x_{1} d y_{1} .
$$

Then $S^{p}$ becomes an $L^{1}\left(\mathbb{R}^{2}\right)$-module. Note that the previous integral exists by Lemma 2.4.

Main Theorem. If $X \in S^{1}, \operatorname{tr}(X)=0$ and $\varepsilon>0$, then there exists $\rho \in L^{1}\left(\mathbb{R}^{2}\right)$ with $\hat{\rho}=1$ on a neighborhood of $(0,0)$ such that

$$
\|\rho \cdot X\|_{S^{1}}<\varepsilon .
$$

Received June 14, 2006; revised June 3, 2008

2000 Mathematics Subject Classification. 43A80, 22E25, 43A45,22E45.

Key words and phrases. Fourier-transform, sobolev, spectral-synthesis.

Based in part on the author's doctoral thesis (University of Chicago) written under the direction of Professor Tim Steger. 
Definition 1.1. If $X \in S^{1}$, the Alpha transform of $X$ is the function on $\mathbb{R}^{2}$ defined by

$$
\alpha(X)(x, y)=\operatorname{tr}\left(T_{x} M_{y} X\right) .
$$

The Alpha transform is related to the module structure on $S^{1}$ in the same way that the classical Fourier transform is related to convolution. In fact, we have the following lemma.

Lemma 1.2. If $q \in L^{1}\left(\mathbb{R}^{2}\right)$ and $X \in S^{1}$, then $\alpha(q \cdot X)=\hat{q} \alpha(X)$.

Proof. Note first that

$$
\begin{aligned}
\alpha\left(\left(x_{1}, y_{1}\right) \cdot X\right)(x, y) & =\operatorname{tr}\left(T_{x} M_{y} T_{y_{1}} M_{-x_{1}} X M_{x_{1}} T_{-y_{1}}\right) \\
& =e^{-2 \pi i y y_{1}} \operatorname{tr}\left(T_{y_{1}} T_{x} M_{-x_{1}} M_{y} X M_{x_{1}} T_{-y_{1}}\right) \\
& =e^{-2 \pi i\left(y y_{1}+x x_{1}\right)} \operatorname{tr}\left(T_{y_{1}} M_{-x_{1}} T_{x} M_{y} X M_{x_{1}} T_{-y_{1}}\right) \\
& =e^{-2 \pi i\left(y y_{1}+x x_{1}\right)} \operatorname{tr}\left(T_{x} M_{y} X\right) \\
& =e^{-2 \pi i\left(y y_{1}+x x_{1}\right)} \alpha(X)(x, y) .
\end{aligned}
$$

The result now follows by integration.

By standard methods, the main theorem leads to the following corollary, which may be viewed as saying that discrete sets are sets of spectral synthesis (more precisely, C-sets) for the Alpha transform.

Main Corollary. Let $D \subseteq \mathbb{R}^{2}$ be a discrete set. Let $\varepsilon>0$. If $X \in S^{1}$ and $\alpha(X)$ vanishes on $D$, then there exists $Y \in S^{1}$ such that $\alpha(Y)$ vanishes on a neighborhood of $D$ and $\|X-Y\|_{S^{1}}<\varepsilon$.

It was shown in [6] (see also [5]) that the spectral synthesis problem for the Alpha transform is closely related to the problem of classifying realizations of the canonical representation (of the Heisenberg group).

\section{Some Lemmas on Integration}

In this section, we prove some slight extensions of [4, Theorem 3.27].

Definition 2.1. Let $f$ be a continuous function defined on $\mathbb{R}^{2}$. We say that $f$ is rapidly decreasing and write $f \in \mathscr{R}\left(\mathbb{R}^{2}\right)$ if for all positive integers $n$, the function $(x, y) \mapsto\left(1+x^{2}+\right.$ $\left.y^{2}\right)^{n} f(x, y)$ is bounded. The best constants in the bounds give a countable family of norms which turn $\mathscr{R}\left(\mathbb{R}^{2}\right)$ into a Frechet space.

Let $\mathscr{T}$ be a Frechet space defined by a countable family of norms.

Definition 2.2. A function $\Phi: \mathbb{R}^{2} \rightarrow \mathscr{T}$ is bounded if for each norm $\sigma$, the function $\sigma \circ \Phi$ is bounded. 
Definition 2.3. A function $\Phi: \mathbb{R}^{2} \rightarrow \mathscr{T}$ is polynomially bounded if for each norm $\sigma$, there exists a polynomial $p$ such that

$$
\sigma \circ \Phi(x, y) \leq p(x, y)
$$

for all $(x, y) \in \mathbb{R}^{2}$.

Lemma 2.4. Let $\Phi: \mathbb{R}^{2} \rightarrow \mathscr{T}$ be a bounded continuous function. Let $f \in L^{1}\left(\mathbb{R}^{2}\right)$. Then

$$
\iint f(x, y) \Phi(x, y) d x d y
$$

exists.

Proof. There is a sequence $\left\{f_{k}\right\}$ of compactly supported continuous functions such that $f_{k} \rightarrow f$ in $L^{1}\left(\mathbb{R}^{2}\right)$. For each $k$, the integral

$$
I_{k}=\iint f_{k}(x, y) \Phi(x, y) d x d y
$$

exists by [4, Theorem 3.27]. For each norm $\sigma$,

$$
\begin{aligned}
\sigma\left(I_{k}-I_{j}\right) & \leq \iint\left|f_{k}(x, y)-f_{j}(x, y)\right| \sigma(\Phi(x, y)) d x d y \\
& \leq \sup \sigma(\Phi(x, y))\left\|f_{k}-f_{j}\right\|_{1} \\
& \rightarrow 0
\end{aligned}
$$

as $k, j \rightarrow \infty$. So $\left\{I_{k}\right\}$ is a Cauchy sequence. Since $\mathscr{T}$ is Frechet, there exists $I \in \mathscr{T}$ such that

$$
\lim _{k \rightarrow \infty} I_{k}=I .
$$

Let $\Lambda$ be a continuous linear functional on $\mathscr{T}$. Then

$$
\begin{aligned}
\Lambda(I) & =\lim _{k \rightarrow \infty} \Lambda\left(I_{k}\right) \\
& =\lim _{k \rightarrow \infty} \Lambda\left(\iint f_{k}(x, y) \Phi(x, y) d x d y\right) \\
& =\lim _{k \rightarrow \infty} \iint f_{k}(x, y) \Lambda(\Phi(x, y)) d x d y \\
& =\iint f(x, y) \Lambda(\Phi(x, y)) d x d y \quad \text { (by the dominated convergence theorem) } \\
& =\iint \Lambda(f(x, y) \Phi(x, y)) d x d y .
\end{aligned}
$$

Therefore, $\iint f(x, y) \Phi(x, y) d x d y=I$.

Lemma 2.5 Let $\Phi: \mathbb{R}^{2} \rightarrow \mathscr{T}$ be a polynomially bounded continuous function. Let $f \in \mathscr{R}\left(\mathbb{R}^{2}\right)$. Then

$$
\iint f(x, y) \Phi(x, y) d x d y
$$


exists.

We omit the proof because it is very similar to the proof of Lemma 2.4.

\section{An Interpolation Theorem}

Theorem 3.1. Let $1 \leq p_{0}, p_{1}, p_{0}^{\prime}, p_{1}^{\prime} \leq \infty$ and suppose that $T: L^{p_{0}}\left(\mathbb{R}^{2}\right) \cap L^{p_{1}}\left(\mathbb{R}^{2}\right) \rightarrow S^{p_{0}^{\prime}} \cap S^{p_{1}^{\prime}}$ is a linear transformation which satisfies

$$
\begin{aligned}
& \|T f\|_{S^{p_{0}^{\prime}}} \leq M_{0}\|f\|_{p_{0}} \quad \text { and } \\
& \|T f\|_{S^{p_{1}^{\prime}}} \leq M_{1}\|f\|_{p_{1}} .
\end{aligned}
$$

Then for each $f \in L^{p_{0}}\left(\mathbb{R}^{2}\right) \cap L^{p_{1}}\left(\mathbb{R}^{2}\right)$ and each $t \in(0,1), T f \in S^{p_{t}^{\prime}}$ and

$$
\|T f\|_{S^{p_{t}^{\prime}}} \leq M_{t}\|f\|_{p_{t}}
$$

where

$$
\begin{aligned}
\frac{1}{p_{t}} & =\frac{t}{p_{1}}+\frac{1-t}{p_{0}}, \\
\frac{1}{p_{t}^{\prime}} & =\frac{t}{p_{1}^{\prime}}+\frac{1-t}{p_{0}^{\prime}} \quad \text { and } \\
M_{t} & =M_{0}^{1-t} M_{1}^{t} .
\end{aligned}
$$

Proof. This follows immediately from the abstract (Calderon-Lions) interpolation theorem once we know that $\left\{L^{p}\left(\mathbb{R}^{2}\right) \mid p \in[1, \infty]\right\}$ and $\left\{S^{p} \mid p \in[1, \infty]\right\}$ form complex interpolation scales. For this, see [2, p38, Example 1 and p44, Proposition 8]. Note that we must take $S^{\infty}$ to be the space of compact operators with the operator norm for all this to work.

\section{Non-commutative Hölder Inequality}

Theorem 4.1. Let $1 \leq p \leq \infty$ and $p^{-1}+p^{\prime-1}=1$. If $A \in S^{p}$ and $B \in S^{p^{\prime}}$, then $A B \in S^{1}$ and

$$
\|A B\|_{S^{1}} \leq\|A\|_{S^{p}}\|B\|_{S^{p^{\prime}}} .
$$

Proof. This follows from Theorem 3.1. For details, see [2].

\section{The Inverse Alpha Transform}

Definition 5.1. Let $f \in L^{1}\left(\mathbb{R}^{2}\right)$. Then the inverse Alpha transform of $f$ is the bounded operator defined by

$$
\Theta(f)=\iint f(x, y) M_{-y} T_{-x} d x d y
$$


Note that this integral exists by Lemma 2.4 .

Lemma 5.2. If $f \in L^{1}\left(\mathbb{R}^{2}\right)$, then for all $g \in L^{2}(\mathbb{R})$,

$$
[\Theta(f) g](v)=\int K(v, w) g(w) d w \quad \text { a.e. }
$$

where

$$
K(\nu, w)=\int f(\nu-w, y) e^{-2 \pi i y v} d y .
$$

Proof. For any $h \in L^{2}(\mathbb{R})$,

$$
\begin{aligned}
\int[\Theta(f) g](v) \overline{h(v)} d v & =\int\left(\iint f(x, y) M_{-y} T_{-x} d x d y g\right)(v) \overline{h(v)} d v \\
& =\iint f(x, y) \int\left(M_{-y} T_{-x} g\right)(v) \overline{h(v)} d v d x d y \\
& =\iint f(x, y) \int e^{-2 \pi i y v} g(v-x) \overline{h(v)} d v d x d y \\
& =\iiint f(x, y) e^{-2 \pi i y v} d y g(v-x) d x \overline{h(v)} d v \\
& =\iiint f(v-w, y) e^{-2 \pi i y v} d y g(w) d w \overline{h(v)} d v \\
& =\iint K(v, w) g(w) d w \overline{h(v)} d v .
\end{aligned}
$$

The application of Fubini's theorem is justified by the fact that $F \in L^{1}\left(\mathbb{R}^{3}\right)$ if

$$
F(x, y, v)=f(x, y) e^{-2 \pi i y v} g(v-x) \overline{h(v)} .
$$

\section{A Minimalist Alpha Inversion Formula}

Lemma 6.1. If $X \in S^{1}$, then

$$
\|\alpha(X)\|_{\infty} \leq\|X\|_{S^{1}} .
$$

Proof. For any $Y \in S^{1}$, we have $|\operatorname{tr}(Y)| \leq \operatorname{tr}(|Y|)$ by the spectral theorem. So for any $X \in S^{1}$,

$$
\begin{aligned}
\|\alpha(X)\|_{\infty} & =\sup _{(x, y) \in \mathbb{R}^{2}}\left|\operatorname{tr}\left(T_{x} M_{y} X\right)\right| \\
& \leq \sup _{(x, y) \in \mathbb{R}^{2}} \operatorname{tr}\left(\left|T_{x} M_{y} X\right|\right) \\
& =\operatorname{tr}(|X|) \quad \text { (since } T_{x} M_{y} \text { is unitary) } \\
& =\|X\|_{S^{1}} .
\end{aligned}
$$


Remark 6.2. Note that if $X$ is given by an integral kernel $K$ of Schwartz class, then

$$
\alpha(X)(x, y)=\int e^{2 \pi i y v} K(\nu, v-x) d v .
$$

Theorem 6.3. If $f$ is a Schwartz class function on $\mathbb{R}^{2}$, then

$$
\alpha(\Theta(f))=f .
$$

Proof. By the Schwartz-Plancherel theorem for the classical Fourier transform, the kernel $K$ of $\Theta(f)$ is of Schwartz class. So

$$
\begin{aligned}
\alpha(\Theta(f))(x, y) & =\int e^{2 \pi i y v} K(v, v-x) d v \\
& =\int e^{2 \pi i y v} \int f\left(x, y^{\prime}\right) e^{-2 \pi i y^{\prime} v} d y^{\prime} d v \\
& =f(x, y) \quad \text { (by the classical Fourier inversion formula.) }
\end{aligned}
$$

Theorem 6.4. If $X \in S^{1}$ is given by an integral kernel $K$ of Schwartz class, then

$$
X=\Theta(\alpha(X))
$$

Proof. By Lemma 5.2, $\Theta(\alpha(X))$ is given by the kernel $\tilde{K}$, where

$$
\tilde{K}\left(v^{\prime}, w\right)=\int \alpha(X)\left(v^{\prime}-w, y\right) e^{-2 \pi i y v^{\prime}} d y .
$$

However, by Remark 6.2 and the classical Fourier inversion formula,

$$
\begin{aligned}
\tilde{K}\left(v^{\prime}, w\right) & =\iint e^{2 \pi i y v} K\left(\nu, v-\left(v^{\prime}-w\right)\right) d v e^{-2 \pi i y v^{\prime}} d y \\
& =K\left(v^{\prime}, w\right)
\end{aligned}
$$

\section{Non-commutative Riemann-Lebesgue Lemma}

Theorem 7.1. If $f \in L^{1}\left(\mathbb{R}^{2}\right)$, then $\|\Theta(f)\|_{\infty} \leq\|f\|_{1}$ and $\Theta(f) \in S^{\infty}$.

Proof. Firstly,

$$
\begin{aligned}
\|\Theta(f)\|_{\infty} & =\left\|\iint f(x, y) M_{-y} T_{-x} d x d y\right\|_{\infty} \\
& \leq \iint|f(x, y)|\left\|M_{-y} T_{-x}\right\|_{\infty} d x d y \\
& =\iint|f(x, y)| d x d y
\end{aligned}
$$




$$
=\|f\|_{1} .
$$

Now, there is a sequence $\left\{f_{k}\right\}$ of compactly supported smooth functions such that $f_{k} \rightarrow f$ in $L^{1}\left(\mathbb{R}^{2}\right)$. Moreover, for each $k$, the operator $\Theta\left(f_{k}\right) \in S^{\infty}$. But, by the previous calculation, $\Theta\left(f_{k}\right) \rightarrow \Theta(f)$ in operator norm. Since $S^{\infty}$ is closed in the operator norm, $\Theta(f) \in S^{\infty}$.

\section{Non-commutative Plancherel Theorem}

Theorem 8.1. $\Theta$ extends to an isometry

$$
\Theta: L^{2}\left(\mathbb{R}^{2}\right) \rightarrow S^{2} .
$$

Proof. Assume first that $f \in L^{1}\left(\mathbb{R}^{2}\right) \cap L^{2}\left(\mathbb{R}^{2}\right)$. Then $\Theta(f)$ is given by the kernel

$$
K(\nu, w)=\int f(\nu-w, y) e^{-2 \pi i y v} d y .
$$

So

$$
\begin{aligned}
\|\Theta(f)\|_{S^{2}}^{2} & =\iint|K(v, w)|^{2} d v d w \\
& =\iint\left|\int f(v-w, y) e^{-2 \pi i y v} d y\right|^{2} d v d w \\
& =\iint|f(u, v)|^{2} d v d u \quad \text { (by the classical Plancherel theorem) } \\
& =\|f\|_{2} .
\end{aligned}
$$

The rest is clear.

\section{Non-commutative Hausdorff-Young Theorem}

Theomre 9.1. Let $1 \leq p \leq 2$ and $p^{-1}+p^{\prime-1}=1$. Then $\Theta$ extends to a bounded operator

$$
\Theta: L^{p}\left(\mathbb{R}^{2}\right) \rightarrow S^{p^{\prime}}
$$

Proof. The endpoint estimates are given by Theorem 7.1 and Theorem 8.1. The result now follows from Theorem 3.1.

\section{An Approximation Lemma}

Recall that if $\varphi, \psi \in L^{2}(\mathbb{R})$, the operator $\varphi \otimes \bar{\psi}: L^{2}(\mathbb{R}) \rightarrow L^{2}(\mathbb{R})$ is defined by

$$
(\varphi \otimes \bar{\psi})(f)=\langle f, \psi\rangle \varphi,
$$


and is of rank one. Thus a general finite rank operator is of the form

$$
X=\sum_{k=1}^{n} \varphi_{k} \otimes \overline{\psi_{k}} \quad \text { with } \varphi_{k}, \psi_{k} \in L^{2}(\mathbb{R}) .
$$

Definition 10.1. The non-commutative Schwartz space is the space $S^{\mathscr{S}}$ of finite rank operators $X: L^{2}(\mathbb{R}) \rightarrow L^{2}(\mathbb{R})$ such that

$$
X=\sum_{k=1}^{n} \varphi_{k} \otimes \overline{\psi_{k}} \quad \text { with } \varphi_{k} \in \mathscr{S},
$$

where $\mathscr{S}$ is the space of Schwartz class functions on $\mathbb{R}$.

Lemma 10.2. Let $X \in S^{1}, \operatorname{tr}(X)=0$ and $\varepsilon>0$. Then there exists $Z \in S^{\mathscr{S}}$ such that $\operatorname{tr}(Z)=0$ and

$$
\|X-Z\|_{S^{1}}<\varepsilon
$$

Proof. It is well known (see e.g. [1]) that there exists a finite rank operator

$$
X_{1}=\sum_{k=1}^{n} \varphi_{k} \otimes \overline{\psi_{k}}
$$

such that $\left\|\psi_{k}\right\|_{2}=1$ and $\left\|X-X_{1}\right\|_{S^{1}}<\frac{\varepsilon}{4}$. It is also well known that there exist $\varphi_{k}^{\prime} \in \mathscr{S}$ such that $\left\|\varphi_{k}-\varphi_{k}^{\prime}\right\|_{2}<\frac{\varepsilon}{4 n}$. Set

$$
X_{2}=\sum_{k=1}^{n} \varphi_{k}^{\prime} \otimes \overline{\psi_{k}} .
$$

Then

$$
\begin{aligned}
\left\|X_{1}-X_{2}\right\| & =\left\|\sum_{k=1}^{n}\left(\varphi_{k}-\varphi_{k}^{\prime}\right) \otimes \overline{\psi_{k}}\right\|_{S^{1}} \\
& \leq \sum_{k=1}^{n}\left\|\varphi_{k}-\varphi_{k}^{\prime}\right\|\left\|_{2}\right\| \psi_{k} \|_{2} \\
& <\sum_{k=1}^{n} \frac{\varepsilon}{4 n} \\
& =\frac{\varepsilon}{4} .
\end{aligned}
$$

Fix $W \in S^{\mathscr{S}}$ such that $\|W\|=\operatorname{tr}(W)=1$ and define $Z=X_{2}-\operatorname{tr}\left(X_{2}\right) W$. Then $Z \in S^{\mathscr{S}}, \operatorname{tr}(Z)=0$ and

$$
\begin{aligned}
\left\|X_{2}-Z\right\|_{S^{1}} & \leq\left|\operatorname{tr}\left(X_{2}\right)\right| \\
& =\left|\sum_{k=1}^{n}\left\langle\varphi_{k}^{\prime}, \psi_{k}\right\rangle\right|
\end{aligned}
$$




$$
\begin{aligned}
& =\left|\sum_{k=1}^{n}\left(\left\langle\varphi_{k}, \psi_{k}\right\rangle-\left\langle\varphi_{k}-\varphi_{k}^{\prime}, \psi_{k}\right\rangle\right)\right| \\
& \leq\left|\sum_{k=1}^{n}\left\langle\varphi_{k}, \psi_{k}\right\rangle\right|+\sum_{k=1}^{n}\left|\left\langle\varphi_{k}-\varphi_{k}^{\prime}, \psi_{k}\right\rangle\right| \\
& \leq\left|\operatorname{tr}\left(X_{1}\right)\right|+\sum_{k=1}^{n}\left\|\varphi_{k}-\varphi_{k}^{\prime}\right\|_{2}\left\|\psi_{k}\right\|_{2} \\
& <\left|\operatorname{tr}\left(X_{1}\right)\right|+\frac{\varepsilon}{4}
\end{aligned}
$$

But

$$
\begin{aligned}
\left|\operatorname{tr}\left(X_{1}\right)\right| & =\left|\operatorname{tr}(X)-\operatorname{tr}\left(X-X_{1}\right)\right| \\
& \leq|\operatorname{tr}(X)|+\left|\operatorname{tr}\left(X-X_{1}\right)\right| \\
& =\left|\operatorname{tr}\left(X-X_{1}\right)\right| \\
& \leq \operatorname{tr}\left(\left|X-X_{1}\right|\right) \\
& =\left\|X-X_{1}\right\|_{S^{1}} \\
& <\frac{\varepsilon}{4} .
\end{aligned}
$$

Therefore, $\left\|X_{2}-Z\right\|_{S^{1}}<\frac{\varepsilon}{2}$. Therefore, $\|X-Z\|<\varepsilon$.

\section{The Action of Differential Operators}

Definition 11.1. For $\varphi \in \mathscr{S}$, define

$$
\begin{aligned}
(P \varphi)(t) & =\frac{d \varphi}{d t} \\
(Q \varphi)(t) & =2 \pi i t \varphi(t) .
\end{aligned}
$$

Lemma 11.2. If $X \in S^{\mathscr{S}}$, then $P X, Q X \in S^{\mathscr{S}}$. Moreover,

$$
\begin{aligned}
& \alpha(P X)=\left(\frac{\partial}{\partial x}-2 \pi i y\right) \alpha(X) \\
& \alpha(Q X)=\frac{\partial}{\partial y} \alpha(X) .
\end{aligned}
$$

Proof. It follows immediately from the definition that $P X, Q X \in S^{\mathscr{S}}$. Since for any $\varphi \in \mathscr{S}$, $\lim _{h \rightarrow 0} \frac{T_{h}-I}{h} \varphi=P \varphi$ in the $L^{2}(\mathbb{R})$ sense, $\lim _{h \rightarrow 0} \frac{T_{h}-I}{h} X=P X$ in $S^{1}$-norm. So

$$
\begin{aligned}
\frac{\partial}{\partial x} \alpha(X)(x, y) & =\frac{\partial}{\partial x} \operatorname{tr}\left(T_{x} M_{y} X\right) \\
& =\lim _{h \rightarrow 0} \frac{\operatorname{tr}\left(T_{x+h} M_{y} X\right)-\operatorname{tr}\left(T_{x} M_{y} X\right)}{h}
\end{aligned}
$$




$$
\begin{aligned}
= & \lim _{h \rightarrow 0} \operatorname{tr}\left(\frac{e^{2 \pi i y h} T_{x} M_{y} T_{h} X-T_{x} M_{y} X}{h}\right) \\
= & \lim _{h \rightarrow 0} \operatorname{tr}\left(\frac{e^{2 \pi i y h} T_{x} M_{y} T_{h} X-e^{2 \pi i y h} T_{x} M_{y} X}{h}\right. \\
& \left.+\frac{e^{2 \pi i y h} T_{x} M_{y} X-T_{x} M_{y} X}{h}\right) \\
= & \lim _{h \rightarrow 0} \operatorname{tr}\left(T_{x} M_{y} \frac{T_{h}-I}{h} X\right)+\frac{e^{2 \pi i y h}-1}{h} \operatorname{tr}\left(T_{x} M_{y} X\right) \\
= & \operatorname{tr}\left(T_{x} M_{y} P X\right)+2 \pi i y \operatorname{tr}\left(T_{x} M_{y} X\right) \\
= & (\alpha(P X)+2 \pi i y \alpha(X))(x, y) .
\end{aligned}
$$

So

$$
\alpha(P X)=\left(\frac{\partial}{\partial x}-2 \pi i y\right) \alpha(X) .
$$

By essentially the same argument, we get

$$
\alpha(Q X)=\frac{\partial}{\partial y} \alpha(X) .
$$

Lemma 11.3. If $X \in S^{\mathscr{S}}$ and $q \in \mathscr{R}\left(\mathbb{R}^{2}\right)$, then $\operatorname{Im}(q \cdot X) \subseteq \mathscr{S}$.

Proof. Without loss of generality, we may assume $X=\varphi \otimes \bar{\psi}$ with $\varphi \in \mathscr{S}$. Let $g \in L^{2}(\mathbb{R})$. Since the map $(x, y) \mapsto\left\langle g, T_{y} M_{-x} \psi\right\rangle$ is bounded and continuous, $q\left(x_{1}, y_{1}\right)\left\langle g, T_{y_{1}} M_{-x_{1}} \psi\right\rangle \in$ $\mathscr{R}\left(\mathbb{R}^{2}\right)$. Since $\left(x_{1}, y_{1}\right) \mapsto T_{y_{1}} M_{-x_{1}} \varphi$ is a polynomially bounded continuous map $\mathbb{R}^{2} \rightarrow \mathscr{S}$,

$$
I=\iint q\left(x_{1}, y_{1}\right)\left\langle g, T_{y_{1}} M_{-x_{1}} \psi\right\rangle T_{y_{1}} M_{-x_{1}} \varphi d x_{1} d y_{1}
$$

exists in $\mathscr{S}$ by Lemma 2.5. Now, for any $h \in L^{2}(\mathbb{R})$,

$$
\begin{aligned}
\int I(v) \overline{h(v)} d v & =\iint q\left(x_{1}, y_{1}\right)\left\langle\left(\left(x_{1}, y_{1}\right) \cdot X\right) g, h\right\rangle d x_{1} d y_{1} \quad \text { (by definition of integral) } \\
& =\langle(q \cdot X) g, h\rangle .
\end{aligned}
$$

It follows that

$$
I=(q \cdot X) g \quad \text { a.e. }
$$

In particular $(q \cdot X) g \in \mathscr{S}$.

Lemma 11.4. Let $X \in S^{\mathscr{S}}$ and $q \in \mathscr{R}\left(\mathbb{R}^{2}\right)$. Then

$$
P(q \cdot X)=\left(-2 \pi i x_{1} q\right) \cdot X+q \cdot(P X) .
$$

In particular, $P(q \cdot X) \in S^{1}$. 
Proof. Since for any $\varphi \in \mathscr{S}$,

$$
\lim _{h \rightarrow 0}\left(\frac{T_{h}-I}{h}\right) \varphi=P \varphi
$$

in the $L^{2}(\mathbb{R})$-sense and $\operatorname{Im}(q \cdot X) \subseteq \mathscr{S}$, we have

$$
P(q \cdot X)=\lim _{h \rightarrow 0} \frac{T_{h}-I}{h}(q \cdot X)
$$

in the strong operator topology. For the same reason,

$$
P X=\lim _{h \rightarrow 0} \frac{T_{h}-I}{h} X
$$

in $S^{1}$-norm. So

$$
\begin{aligned}
\lim _{h \rightarrow 0} \frac{T_{h}-I}{h}(q \cdot X)= & \lim _{h \rightarrow 0} \frac{T_{h}-I}{h} \iint q\left(x_{1}, y_{1}\right)\left(x_{1}, y_{1}\right) \cdot X d x_{1} d y_{1} \\
= & \lim _{h \rightarrow 0} \iint q\left(x_{1}, y_{1}\right) \frac{T_{h}-I}{h}\left(x_{1}, y_{1}\right) \cdot X d x_{1} d y_{1} \\
= & \lim _{h \rightarrow 0} \iint q\left(x_{1}, y_{1}\right) \frac{1}{h}\left(e^{-2 \pi i x_{1} h} T_{y_{1}} M_{-x_{1}} T_{h} X M_{x_{1}} T_{-y_{1}}\right. \\
& \left.-T_{y_{1}} M_{-x_{1}} X M_{x_{1}} T_{-y_{1}}\right) d x_{1} d y_{1} \\
= & \lim _{h \rightarrow 0} \iint q\left(x_{1}, y_{1}\right)\left(\frac{e^{-2 \pi i x_{1} h}-1}{h} T_{y_{1}} M_{-x_{1}} T_{h} X M_{x_{1}} T_{-y_{1}}\right. \\
& \left.+T_{y_{1}} M_{-x_{1}} \frac{T_{h}-I}{h} X M_{x_{1}} T_{-y_{1}}\right) d x_{1} d y_{1} \\
= & \iint q\left(x_{1}, y_{1}\right)\left(-2 \pi i x_{1}\left(x_{1}, y_{1}\right) \cdot X+\left(x_{1}, y_{1}\right) \cdot(P X)\right) d x_{1} d y_{1} \\
= & \left(-2 \pi i x_{1} q\right) \cdot X+q \cdot(P X)
\end{aligned}
$$

in $S^{1}$-norm. This proves the claim.

Lemma 11.5. Let $X \in S^{\mathscr{S}}$ and $q \in \mathscr{R}\left(\mathbb{R}^{2}\right)$. Then

$$
Q(q \cdot X)=\left(-2 \pi i y_{1} q\right) \cdot X+q \cdot(Q X)
$$

In particular, $Q(q \cdot X) \in S^{1}$.

Proof. This is proved by the same sort of reasoning as Lemma 11.4.

Lemma 11.6. If $X \in S^{\mathscr{S}}$ and $q \in \mathscr{R}\left(\mathbb{R}^{2}\right)$, then

$$
\alpha(P(q \cdot X))=\left(\frac{\partial}{\partial x}-2 \pi i y\right) \alpha(q \cdot X) .
$$

Proof. By Lemma 11.4, we have

$$
P(q \cdot X)=\left(-2 \pi i x_{1} q\right) \cdot X+q \cdot(P X) .
$$


It follows that

$$
\begin{aligned}
\alpha(P(q \cdot X)) & =\frac{\partial \hat{q}}{\partial x} \alpha(X)+\hat{q} \alpha(P X) \\
& =\frac{\partial \hat{q}}{\partial x} \alpha(X)+\hat{q}\left(\frac{\partial}{\partial x}-2 \pi i y\right) \alpha(X) \\
& =\frac{\partial}{\partial x}(\hat{q} \alpha(X))-2 \pi i y \hat{q} \alpha(X) \\
& =\left(\frac{\partial}{\partial x}-2 \pi i y\right)(\hat{q} \alpha(X)) \\
& =\left(\frac{\partial}{\partial x}-2 \pi i y\right) \alpha(q \cdot X)
\end{aligned}
$$

Lemma 11.7. If $X \in S^{\mathscr{S}}$ and $q \in \mathscr{R}\left(\mathbb{R}^{2}\right)$, then

$$
\alpha(Q(q \cdot X))=\frac{\partial}{\partial y} \alpha(q \cdot X) .
$$

Proof. This is proved in the same way as Lemma 11.6.

\section{The Harmonic Oscillator}

Definition 12.1. The harmonic oscillator is the differential operator

$$
H=P^{2}+Q^{2} \text {. }
$$

Lemma 12.2. If $X \in S^{\mathscr{S}}$ and $q \in \mathscr{R}\left(\mathbb{R}^{2}\right)$, then $H(q \cdot X) \in S^{1}$ and

$$
\alpha(H(q \cdot X))=\mathscr{D} \alpha(q \cdot X),
$$

where

$$
\mathscr{D}=\left(\frac{\partial}{\partial x}-2 \pi i y\right)^{2}+\left(\frac{\partial}{\partial y}\right)^{2}
$$

Proof. By Lemma 11.4 and Lemma 11.5, we have

$$
\begin{aligned}
& P^{2}(q \cdot X)=P\left(\left(-2 \pi i x_{1} q\right) \cdot X\right)+P(q \cdot(P X)) \quad \text { and } \\
& Q^{2}(q \cdot X)=Q\left(\left(-2 \pi i y_{1} q\right) \cdot X\right)+Q(q \cdot(Q X)) .
\end{aligned}
$$

So $P^{2}(q \cdot X), Q^{2}(q \cdot X) \in S^{1}$ and hence $H(q \cdot X) \in S^{1}$.

By Lemma 11.6 and Lemma 11.7, we have

$$
\alpha\left(P^{2}(q \cdot X)\right)=\alpha\left(P\left(\left(-2 \pi i x_{1} q\right) \cdot X\right)\right)+\alpha(P(q \cdot(P X)))
$$




$$
\begin{aligned}
& =\left(\frac{\partial}{\partial x}-2 \pi i y\right) \alpha\left(\left(-2 \pi i x_{1} q\right) \cdot X\right)+\left(\frac{\partial}{\partial x}-2 \pi i y\right) \alpha(q \cdot(P X)) \\
& =\left(\frac{\partial}{\partial x}-2 \pi i y\right)\left(\alpha\left(\left(-2 \pi i x_{1} q\right) \cdot X\right)+\alpha(q \cdot(P X))\right) \\
& =\left(\frac{\partial}{\partial x}-2 \pi i y\right)\left(\frac{\partial \hat{q}}{\partial x} \alpha(X)+\hat{q}\left(\frac{\partial}{\partial x}-2 \pi i y\right) \alpha(X)\right) \\
& =\left(\frac{\partial}{\partial x}-2 \pi i y\right)\left(\frac{\partial}{\partial x}(\hat{q} \alpha(X))-2 \pi i y \alpha(X)\right) \\
& =\left(\frac{\partial}{\partial x}-2 \pi i y\right)^{2}(\hat{q} \alpha(X)) \\
& =\left(\frac{\partial}{\partial x}-2 \pi i y\right)^{2} \alpha(q \cdot X) .
\end{aligned}
$$

and

$$
\begin{aligned}
\alpha\left(Q^{2}(q \cdot X)\right) & =\frac{\partial}{\partial y} \alpha\left(\left(-2 \pi i y_{1} q\right) \cdot X\right)+\frac{\partial}{\partial y} \alpha(q \cdot(Q X)) \\
& =\frac{\partial}{\partial y}\left(\alpha\left(\left(-2 \pi i y_{1} q\right) \cdot X\right)+\alpha(q \cdot(Q X))\right) \\
& =\frac{\partial}{\partial y}\left(\frac{\partial \hat{q}}{\partial y} \alpha(X)+\hat{q} \frac{\partial}{\partial y} \alpha(X)\right) \\
& =\frac{\partial^{2}}{\partial y^{2}}(\hat{q} \alpha(X)) \\
& =\frac{\partial^{2}}{\partial y^{2}} \alpha(q \cdot X) .
\end{aligned}
$$

The result now follows.

Theorem 12.3. There is a complete orthonormal set $\left\{\varphi_{k}\right\}$ in $L^{2}(\mathbb{R})$ such that

$$
H \varphi_{k}=-2 \pi(2 k+1) \varphi_{k}
$$

Proof. See [3, Lemma 10.34].

Corollary 12.4. For $p>1$,

$$
H^{-1} \in S^{p}
$$

Proof. This follows from the $p$-series test.

\section{A Versal Constant}

For $\delta>0$, let $B_{\delta}=\left\{(x, y) \in \mathbb{R}^{2}: \sqrt{x^{2}+y^{2}}<\delta\right\}$. Fix a radial smooth function $\tau$ with support in $B_{1}$ and identically 1 in a neighborhood of 0 . Set

$$
V=\|\check{\tau}\|_{1} .
$$


Set

$$
\tau_{\delta}(x, y)=\tau(x / \delta, y / \delta) .
$$

Then

$$
\begin{aligned}
\check{\tau}_{\delta}\left(x_{1}, y_{1}\right) & =\iint e^{2 \pi i\left(x x_{1}+y y_{1}\right)} \tau_{\delta}(x, y) d x d y \\
& =\iint e^{2 \pi i\left(x x_{1}+y y_{1}\right)} \tau(x / \delta, y / \delta) d x d y \\
& =\iint e^{2 \pi i \delta\left(x x_{1}+y y_{1}\right)} \tau(x, y) \delta^{2} d x d y \\
& =\delta^{2} \check{\tau}\left(\delta x_{1}, \delta y_{1}\right) .
\end{aligned}
$$

So for any $\delta>0$,

$$
\begin{aligned}
\left\|\check{\tau}_{\delta}\right\|_{1} & =\delta^{2} \iint\left|\check{\tau}\left(\delta x_{1}, \delta y_{1}\right)\right| d x_{1} d y_{1} \\
& =\delta^{2} \iint\left|\check{\tau}\left(x_{1}, y_{1}\right)\right| \delta^{-2} d x_{1} d y_{1} \\
& =\|\check{\tau}\|_{1} \\
& =V .
\end{aligned}
$$




\section{The Heart of the Matter}

14.1. If $X \in S^{\mathscr{S}}, \operatorname{tr}(X)=0$ and $\varepsilon>0$, then there exists $\rho \in L^{1}\left(\mathbb{R}^{2}\right)$ with $\hat{\rho}=1$ on a neighborhood of $(0,0)$ such that

$$
\|\rho \cdot X\|_{S_{1}}<\varepsilon
$$

Proof. Let $r=\sqrt{x^{2}+y^{2}}$. Let

$$
C_{1}=\sup _{B_{1}}|\nabla \alpha(X)|
$$

Since $\alpha(X)$ is smooth and $\alpha(X)(0,0)=0$, it follows from the mean value theorem that

$$
|\alpha(X)(x, y)| \leq C_{1} r \quad \text { on } B_{1} .
$$

Let

$$
\begin{aligned}
& C_{2}=\sup _{B_{1}}|\Delta \alpha(X)| \\
& D_{1}=\sup _{B_{1}}|\tau| \\
& D_{2}=\sup _{B_{1}}|\nabla \tau| \\
& D_{3}=\sup _{B_{1}}|\Delta \tau| .
\end{aligned}
$$

Then

$$
\begin{aligned}
\mathscr{D}\left(\tau_{\delta} \alpha(X)\right)= & \left(\Delta-4 \pi i y \frac{\partial}{\partial x}-4 \pi^{2} y^{2}\right)\left(\tau_{\delta} \alpha(X)\right) \\
= & \left(\Delta \tau_{\delta}\right) \alpha(X)+2 \nabla \tau_{\delta} \cdot \nabla \alpha(X)+\tau_{\delta} \Delta \alpha(X) \\
& -4 \pi i y\left(\frac{\partial \tau_{\delta}}{\partial x} \alpha(X)+\tau_{\delta} \frac{\partial}{\partial x} \alpha(X)\right) \\
& -4 \pi^{2} y^{2} \tau_{\delta} \alpha(X) .
\end{aligned}
$$

Therefore, on $B_{\delta}$, we have by the Cauchy-Schwarz inequality that

$$
\begin{aligned}
\left|\mathscr{D}\left(\tau_{\delta} \alpha(X)\right)\right| \leq & {\left[\left|\Delta \tau_{\delta}\right||\alpha(X)|+2\left|\nabla \tau_{\delta}\right||\nabla \alpha(X)|+\left|\tau_{\delta}\right||\Delta \alpha(X)|\right.} \\
& +4 \pi|y|\left(\left|\frac{\partial \tau_{\delta}}{\partial x}\right||\alpha(X)|+\left|\tau_{\delta}\right|\left|\frac{\partial}{\partial x} \alpha(X)\right|\right) \\
& \left.+4 \pi^{2}|y|^{2}\left|\tau_{\delta}\right||\alpha(X)|\right] \\
\leq & \left(\left|\Delta \tau_{\delta}\right||\alpha(X)|+2\left|\nabla \tau_{\delta}\right||\nabla \alpha(X)|+\left|\tau_{\delta}\right||\Delta \alpha(X)|\right. \\
& \left.+4 \pi r\left(\left|\nabla \tau_{\delta}\right||\alpha(X)|+\left|\tau_{\delta}\right||\nabla \alpha(X)|\right)+4 \pi^{2} r^{2}\left|\tau_{\delta}\right||\alpha(X)|\right) \\
\leq & \left(\delta^{-2} D_{3} C_{1} r+2 \delta^{-1} D_{2} C_{1}+D_{1} C_{2}\right. \\
& \left.+4 \pi r\left(\delta^{-1} D_{2} C_{1} r+D_{1} C_{1}\right)+4 \pi^{2} r^{2} D_{1} C_{1} r\right) \\
\leq & \left(\delta^{-2} D_{3} C_{1} r+\delta^{-1}\left(2 D_{2} C_{1}+4 \pi D_{2} C_{1}\right)+\left(D_{1} C_{2}+4 \pi D_{1} C_{1}+4 \pi^{2} D_{1} C_{1}\right)\right)
\end{aligned}
$$




$$
\leq\left(A_{1} \delta^{-2} r+A_{2} \delta^{-1}+A_{3}\right)
$$

Moreover, $\mathscr{D}\left(\tau_{\delta} \alpha(X)\right)$ is supported in $B_{\delta}$. Fix $p \in(1,2)$. Then

$$
\begin{aligned}
\left\|\mathscr{D}\left(\tau_{\delta} \alpha(X)\right)\right\|_{p} \leq & A_{1} \delta^{-2}\left(\iint_{B_{\delta}} r^{p} d x d y\right)^{1 / p}+A_{2} \delta^{-1}\left(\iint_{B_{\delta}} d x d y\right)^{1 / p} \\
& +A_{3}\left(\iint_{B_{\delta}} d x d y\right)^{1 / p} \\
\leq & (2 \pi)^{1 / p}\left(A_{1} \delta^{-2}\left(\frac{\delta^{p+2}}{p+2}\right)^{1 / p}+A_{2} \delta^{-1}\left(\frac{\delta^{2}}{2}\right)^{1 / p}+A_{3}\left(\frac{\delta^{2}}{2}\right)^{1 / p}\right) \\
= & (2 \pi)^{1 / p}\left(A_{1}(p+2)^{-1 / p}+A_{2} 2^{-1 / p}\right) \delta^{2 / p-1}+A_{3} 2^{-1 / p} \delta^{2 / p} .
\end{aligned}
$$

Therefore,

$$
\lim _{\delta \rightarrow 0} \mathscr{D}\left(\tau_{\delta} \alpha(X)\right)=0 \quad \text { in } L^{p}\left(\mathbb{R}^{2}\right) .
$$

If $p^{-1}+p^{-1}=1$, then we have

$$
\begin{array}{rlrl}
\lim _{\delta \rightarrow 0} H\left(\check{\tau}_{\delta} \cdot X\right) & =\lim _{\delta \rightarrow 0} \Theta\left(\mathscr{D}\left(\tau_{\delta} \alpha(X)\right)\right) \quad \text { (by Theorem 6.4 and Lemma 12.2) } \\
& =\Theta\left(\lim _{\delta \rightarrow 0} \mathscr{D}\left(\tau_{\delta} \alpha(X)\right)\right) & & \text { (by Theorem 9.1) } \\
& =0 \quad \text { in } S^{p^{\prime}} . &
\end{array}
$$

By Corollary $12.4, H^{-1} \in S^{p}$. So by Theorem 4.1, we have

$$
\begin{aligned}
\lim _{\delta \rightarrow 0} \check{\tau}_{\delta} \cdot X & =H^{-1}\left(\lim _{\delta \rightarrow 0} H\left(\check{\tau}_{\delta} \cdot X\right)\right) \\
& =0 \quad \text { in } S^{1} .
\end{aligned}
$$

Therefore, there exists $\delta_{0}>0$ such that $\left\|\check{\tau}_{\delta_{0}} \cdot X\right\|_{S^{1}}<\varepsilon$. Take $\rho=\check{\tau}_{\delta_{0}}$. Then $\hat{\rho}=1$ in a neighborhood of $(0,0)$ and $\|\rho \cdot X\|_{S^{1}}<\varepsilon$.

Proof. of the Main Theorem. Now, if $X \in S^{1}$ and $\operatorname{tr}(X)=0$, by Theorem 10.2 we can find $X^{\prime} \in S^{\mathscr{S}}$ such that $\left\|X-X^{\prime}\right\|_{S^{1}}<\frac{\varepsilon}{2 V}$ and $\operatorname{tr}\left(X^{\prime}\right)=0$. Then by Lemma 14.1 we can find $\rho \in L^{1}\left(\mathbb{R}^{2}\right)$ such that $\left\|\rho \cdot X^{\prime}\right\|_{S^{1}}<\frac{\varepsilon}{2}$. Thus

$$
\begin{aligned}
\|\rho \cdot X\|_{S^{1}} & =\left\|\rho \cdot\left[X^{\prime}-\left(X^{\prime}-X\right)\right]\right\|_{S^{1}} \\
& \leq\left\|\rho \cdot X^{\prime}\right\|_{S^{1}}+\left\|\rho \cdot\left(X^{\prime}-X\right)\right\|_{S^{1}} \\
& \leq \frac{\varepsilon}{2}+\|\rho\|_{1}\left\|X-X^{\prime}\right\|_{S^{1}} \\
& <\varepsilon
\end{aligned}
$$




\section{References}

[1] M. Reed and B. Simon, Methods of Modern Mathematical Physics I: Functional Analysis, Academic Press, New York, 1972.

[2] _ Methods of Modern Mathematical Physics II: Fourier Analysis, Self-Adjointness, Academic Press, New York, 1975.

[3] John Roe, Elliptic operators, topology and asymptotic methods, Longman Scientific and Technical, Harlow, Essex, England, 1988.

[4] Walter Rudin, Functional Analysis, McGraw-Hill, Inc., New York, 1991.

[5] M. K. Vemuri, Realizations of the canonical representation, Ph.D. thesis, Univ. of Chicago, 1997.

[6] _ Realizations of the canonical representation, Proc. Indian Acad. Sci. Math. Sci. 118(2008), $115-131$.

Chennai Mathematical Institute, SIPCOT Information Technology Park, Navalur Post, SIRUSERI 603 103, India.

E-mail: mkvemuri@cmi.ac.in 